\title{
An Application of a Poisson Distribution Series on Certain Analytic Functions
}

\author{
Saurabh Porwal \\ Department of Mathematics, U.I.E.T., C.S.J.M. University, Kanpur, Uttar Pradesh 208024, India \\ Correspondence should be addressed to Saurabh Porwal; saurabhjcb@rediffmail.com
}

Received 25 November 2013; Accepted 7 January 2014; Published 18 February 2014

Academic Editor: Janne Heittokangas

Copyright (C) 2014 Saurabh Porwal. This is an open access article distributed under the Creative Commons Attribution License, which permits unrestricted use, distribution, and reproduction in any medium, provided the original work is properly cited.

The purpose of the present paper is to introduce a Poisson distribution series and obtain necessary and sufficient conditions for this series belonging to the classes $T(\lambda, \alpha)$ and $C(\lambda, \alpha)$. We also consider an integral operator related to this series.

\section{Introduction}

Let $A$ denote the class of functions $f$ of the form

$$
f(z)=z+\sum_{n=2}^{\infty} a_{n} z^{n}
$$

which are analytic in the open unit disk $U=\{z: z \in$ $C$ and $|z|<1\}$ and satisfy the normalization condition $f(0)=f^{\prime}(0)-1=0$. Further, we denote by $S$ the subclass of $A$ consisting of functions of the form (1) which are also univalent in $U$ and let $T$ be the subclass of $S$ consisting of functions of the form

$$
f(z)=z-\sum_{n=2}^{\infty}\left|a_{n}\right| z^{n} .
$$

Let $T(\lambda, \alpha)$ be the subclass of $T$ consisting of functions which satisfy the condition

$$
\operatorname{Re}\left\{\frac{z f^{\prime}(z)}{\lambda z f^{\prime}(z)+(1-\lambda) f(z)}\right\}>\alpha,
$$

for some $\alpha(0 \leq \alpha<1), \lambda(0 \leq \lambda<1)$ and for all $z \in U$.

Also, we let $C(\lambda, \alpha)$ denote the subclass of $T$ consisting of functions which satisfy the condition

$$
\operatorname{Re}\left\{\frac{f^{\prime}(z)+z f^{\prime \prime}(z)}{f^{\prime}(z)+\lambda z f^{\prime \prime}(z)}\right\}>\alpha,
$$

for some $\alpha(0 \leq \alpha<1), \lambda(0 \leq \lambda<1)$ and for all $z \in U$.
From (3) and (4) it is easy to verify that

$$
f(z) \in C(\lambda, \alpha) \Longleftrightarrow z f^{\prime}(z) \in T(\lambda, \alpha) .
$$

The classes $T(\lambda, \alpha)$ and $C(\lambda, \alpha)$ were extensively studied by Altintas and Owa [1] and certain conditions for hypergeometric functions and generalized Bessel functions for these classes were studied by Mostafa [2] and Porwal and Dixit [3].

It is worthy to note that $T(0, \alpha) \equiv T^{*}(\alpha)$, the class of starlike functions of order $\alpha(0 \leq \alpha<1)$ and $C(0, \alpha) \equiv C(\alpha)$, the class of convex functions of order $\alpha(0 \leq \alpha<1)$ (see [4]).

A variable $x$ is said to have Poisson distribution if it takes the values $0,1,2,3, \ldots$ with probabilities $e^{-m}$, $m e^{-m} / 1 !, m^{2} e^{-m} / 2 !, m^{3} e^{-m} / 3 !, \ldots$, respectively, where $m$ is called the parameter.

Thus

$$
P(x=k)=\frac{m^{k} e^{-m}}{k !}, \quad k=0,1,2,3, \ldots
$$

Now, we introduce a power series whose coefficients are probabilities of the Poisson distribution:

$$
K(m, z)=z+\sum_{n=2}^{\infty} \frac{m^{n-1}}{(n-1) !} e^{-m} z^{n} .
$$

We note that, by ratio test, the radius of convergence of the above series is infinity.

Now, we introduce the series

$$
F(m, z)=2 z-K(m, z)=z-\sum_{n=2}^{\infty} \frac{m^{n-1}}{(n-1) !} e^{-m} z^{n} .
$$


Motivated by results on connections between various subclasses of analytic univalent functions by using hypergeometric functions (see [5-10]) and generalized Bessel functions (see $[3,11-13])$, we obtain necessary and sufficient conditions for function $F(m, z)$ belonging to the classes $T(\lambda, \alpha)$ and $C(\lambda, \alpha)$. Finally, we give conditions for an integral operator $G(m, z)$ belonging to the classes $T(\lambda, \alpha)$ and $C(\lambda, \alpha)$.

\section{Main Results}

To establish our main results, we will require the following Lemmas according to Altintas and Owa [1].

Lemma 1 (see [1]). A function $f(z)$ defined by (2) is in the class $T(\lambda, \alpha)$ if and only if

$$
\sum_{n=2}^{\infty}[n-\lambda \alpha n-\alpha+\lambda \alpha]\left|a_{n}\right| \leq 1-\alpha .
$$

Lemma 2 (see [1]). A function $f(z)$ defined by (2) is in the class $C(\lambda, \alpha)$ if and only if

$$
\sum_{n=2}^{\infty} n[n-\lambda \alpha n-\alpha+\lambda \alpha]\left|a_{n}\right| \leq 1-\alpha .
$$

Theorem 3. If $m>0$, then $F(m, z)$ is in $T(\lambda, \alpha)$, if and only if

$$
m e^{m}(1-\alpha \lambda) \leq 1-\alpha \text {. }
$$

Proof. Since

$$
F(m, z)=z-\sum_{n=2}^{\infty} \frac{e^{-m} m^{n-1}}{(n-1) !} z^{n},
$$

according to Lemma 1, we must show that

$$
\sum_{n=2}^{\infty}[n(1-\lambda \alpha)-\alpha(1-\lambda)] \frac{e^{-m} m^{n-1}}{(n-1) !} \leq 1-\alpha .
$$

Now

$$
\begin{aligned}
\sum_{n=2}^{\infty} & {[n(1-\alpha \lambda)-\alpha(1-\lambda)] \frac{e^{-m} m^{n-1}}{(n-1) !} } \\
& =e^{-m}\left[\sum_{n=2}^{\infty}[(n-1)(1-\alpha \lambda)+(1-\alpha)] \frac{m^{n-1}}{(n-1) !}\right] \\
& =e^{-m}\left[(1-\alpha \lambda) \sum_{n=2}^{\infty} \frac{m^{n-1}}{(n-2) !}+(1-\alpha) \sum_{n=2}^{\infty} \frac{m^{n-1}}{(n-1) !}\right] \\
& =e^{-m}\left[(1-\alpha \lambda) m \sum_{n=0}^{\infty} \frac{m^{n}}{n !}+(1-\alpha) \sum_{n=1}^{\infty} \frac{m^{n}}{n !}\right] \\
& =e^{-m}\left[(1-\alpha \lambda) m e^{m}+(1-\alpha)\left(e^{m}-1\right)\right] \\
& =(1-\alpha \lambda) m+(1-\alpha)\left(1-e^{-m}\right) .
\end{aligned}
$$

But this last expression is bounded previously by $1-\alpha$ if and only if (11) holds.

Thus the proof of Theorem 3 is established.
Theorem 4. If $m>0$, then $F(m, z)$ is in $C(\lambda, \alpha)$, if and only if

$$
e^{m}\left[(1-\alpha \lambda) m^{2}+(3-2 \alpha \lambda-\alpha) m\right] \leq 1-\alpha
$$

Proof. Since

$$
F(m, z)=z-\sum_{n=2}^{\infty} \frac{e^{-m} m^{n-1}}{(n-1) !} z^{n},
$$

according to Lemma 2, we must show that

$$
\sum_{n=2}^{\infty} n[n(1-\lambda \alpha)-\alpha(1-\lambda)] \frac{e^{-m} m^{n-1}}{(n-1) !} \leq 1-\alpha .
$$

Now

$$
\begin{aligned}
& \sum_{n=2}^{\infty} n[n(1-\alpha \lambda)-\alpha(1-\lambda)] \frac{e^{-m} m^{n-1}}{(n-1) !} \\
&=\sum_{n=2}^{\infty}\{(1-\alpha \lambda)(n-1)(n-2) \\
&\quad+(3-2 \alpha \lambda-\alpha)(n-1)+(1-\alpha)\} \frac{e^{-m} m^{n-1}}{(n-1) !} \\
&=e^{-m}\left[(1-\alpha \lambda) \sum_{n=3}^{\infty} \frac{m^{n-1}}{(n-3) !}+(3-2 \alpha \lambda-\alpha)\right. \\
& \quad \times \sum_{n=2}^{\infty} \frac{m^{n-1}(n-2) !}{\left(n-(1-\alpha) \sum_{n=2}^{\infty} \frac{m^{n-1}}{(n-1) !}\right]} \\
&=e^{-m}\left[(1-\alpha \lambda) m^{2} e^{m}+(3-2 \alpha \lambda-\alpha) m e^{m}\right. \\
&\left.\quad+(1-\alpha)\left(e^{m}-1\right)\right] \\
&=(1-\alpha \lambda) m^{2}+(3-2 \alpha \lambda-\alpha) m+(1-\alpha)\left(1-e^{-m}\right) .
\end{aligned}
$$

But this last expression is bounded above by $1-\alpha$ if and only if (15) holds. This completes the proof of Theorem 4.

\section{An Integral Operator}

In the following theorem, we obtain similar results in connection with a particular integral operator $G(m, z)$ as follows:

$$
G(m, z)=\int_{0}^{z} \frac{F(m, t)}{t} d t
$$

Theorem 5. If $m>0$, then $G(m, z)$ defined by (19) is in $C(\lambda, \alpha)$ if and only if

$$
m e^{m}(1-\alpha \lambda) \leq 1-\alpha
$$

Proof. Since

$$
\begin{aligned}
G(m, z) & =z-\sum_{n=2}^{\infty} \frac{e^{-m} m^{n-1}}{(n-1) !} \frac{z^{n}}{n} \\
& =z-\sum_{n=2}^{\infty} \frac{e^{-m} m^{n-1}}{n !} z^{n}
\end{aligned}
$$


by Lemma 2, we need only to show that

$$
\sum_{n=2}^{\infty} n[n(1-\lambda \alpha)-\alpha(1-\lambda)] \frac{e^{-m} m^{n-1}}{n !} \leq 1-\alpha .
$$

Now

$$
\begin{aligned}
\sum_{n=2}^{\infty} n & {[n(1-\lambda \alpha)-\alpha(1-\lambda)] \frac{e^{-m} m^{n-1}}{n !} } \\
& =\sum_{n=2}^{\infty}[n(1-\lambda \alpha)-\alpha(1-\lambda)] \frac{e^{-m} m^{n-1}}{(n-1) !} \\
& =e^{-m}\left[\sum_{n=2}^{\infty}[(n-1)(1-\alpha \lambda)+(1-\alpha)] \frac{m^{n-1}}{(n-1) !}\right] \\
& =e^{-m}\left[(1-\alpha \lambda) \sum_{n=2}^{\infty} \frac{m^{n-1}}{(n-2) !}+(1-\alpha) \sum_{n=2}^{\infty} \frac{m^{n-1}}{(n-1) !}\right] \\
& =e^{-m}\left[(1-\alpha \lambda) m \sum_{n=0}^{\infty} \frac{m^{n}}{n !}+(1-\alpha) \sum_{n=1}^{\infty} \frac{m^{n}}{n !}\right] \\
& =e^{-m}\left[(1-\alpha \lambda) m e^{m}+(1-\alpha)\left(e^{m}-1\right)\right] \\
& =(1-\alpha \lambda) m+(1-\alpha)\left(1-e^{-m}\right),
\end{aligned}
$$

which is bounded above by $1-\alpha$, if and only if (20) holds.

Theorem 6. If $m>0$, then $G(m, z)$ defined by (19) is in $T(\lambda, \alpha)$ if and only if

$$
\left[(1-\alpha \lambda)-\frac{\alpha(1-\lambda)}{m}\right]\left(1-e^{-m}\right)+\alpha(1-\lambda) e^{-m} \leq 1-\alpha .
$$

Proof. The proof of this theorem is similar to that of Theorem 5. Therefore we omit the details involved.

\section{Conflict of Interests}

The author declares that there is no conflict of interests regarding the publication of this paper.

\section{References}

[1] O. Altintas and S. Owa, "On subclasses of univalent functions with negative coefficients," Pusan Kyongnam Mathematical Journal, vol. 4, pp. 41-56, 1988.

[2] A. O. Mostafa, "A study on starlike and convex properties for hypergeometric functions," Journal of Inequalities in Pure and Applied Mathematics, vol. 10, no. 3, article 87, pp. 1-16, 2009.

[3] S. Porwal and K. K. Dixit, "An application of generalized Bessel functions on certain analytic functions," Acta Universitatis Matthiae Belii. Series Mathematics, pp. 51-57, 2013.

[4] H. Silverman, "Univalent functions with negative coefficients," Proceedings of the American Mathematical Society, vol. 51, pp. 109-116, 1975.
[5] B. C. Carlson and D. B. Shaffer, "Starlike and prestarlike hypergeometric functions," SIAM Journal on Mathematical Analysis, vol. 15, no. 4, pp. 737-745, 1984.

[6] N. E. Cho, S. Y. Woo, and S. Owa, "Uniform convexity properties for hypergeometric functions," Fractional Calculus \& Applied Analysis for Theory and Applications, vol. 5, no. 3, pp. 303-313, 2002.

[7] E. P. Merkes and W. T. Scott, "Starlike hypergeometric functions," Proceedings of the American Mathematical Society, vol. 12, pp. 885-888, 1961.

[8] S. Porwal and K. K. Dixit, "An application of certain convolution operator involving hypergeometric functions," Journal of Rajasthan Academy of Physical Sciences, vol. 9, no. 2, pp. 173186,2010

[9] A. K. Sharma, S. Porwal, and K. K. Dixit, "Class mappings properties of convolutions involving certain univalent functions associated with hypergeometric functions," Electronic Journal of Mathematical Analysis and Applications, vol. 1, no. 2, pp. 326333, 2013.

[10] A. Gangadharan, T. N. Shanmugam, and H. M. Srivastava, "Generalized hypergeometric functions associated with $k$ uniformly convex functions," Computers \& Mathematics with Applications, vol. 44, no. 12, pp. 1515-1526, 2002.

[11] A. Baricz, Generalized Bessel Functions of the First Kind, vol. 1994 of Lecture Notes in Mathematics, Springer, Berlin, Germany, 2010.

[12] S. R. Mondal and A. Swaminathan, "Geometric properties of generalized Bessel functions," Bulletin of the Malaysian Mathematical Sciences Society, vol. 35, no. 1, pp. 179-194, 2012.

[13] S. Porwal, "Mapping properties of generalized Bessel functions on some subclasses of univalent functions," Analele Universitatii Oradea Fasc. Matematica, vol. 20, no. 2, pp. 51-60, 2013. 


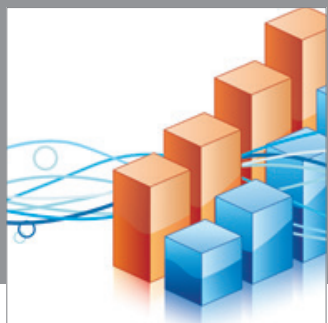

Advances in

Operations Research

mansans

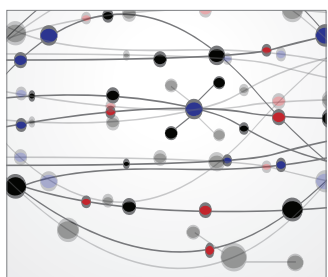

The Scientific World Journal
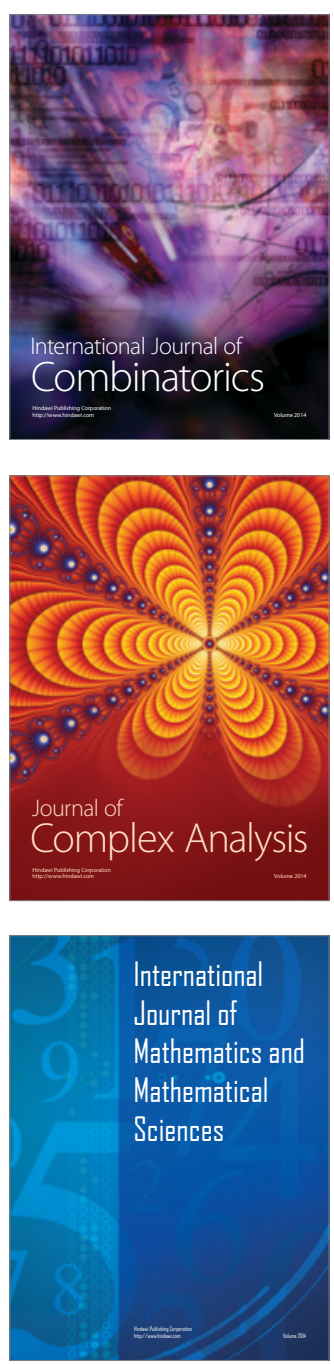
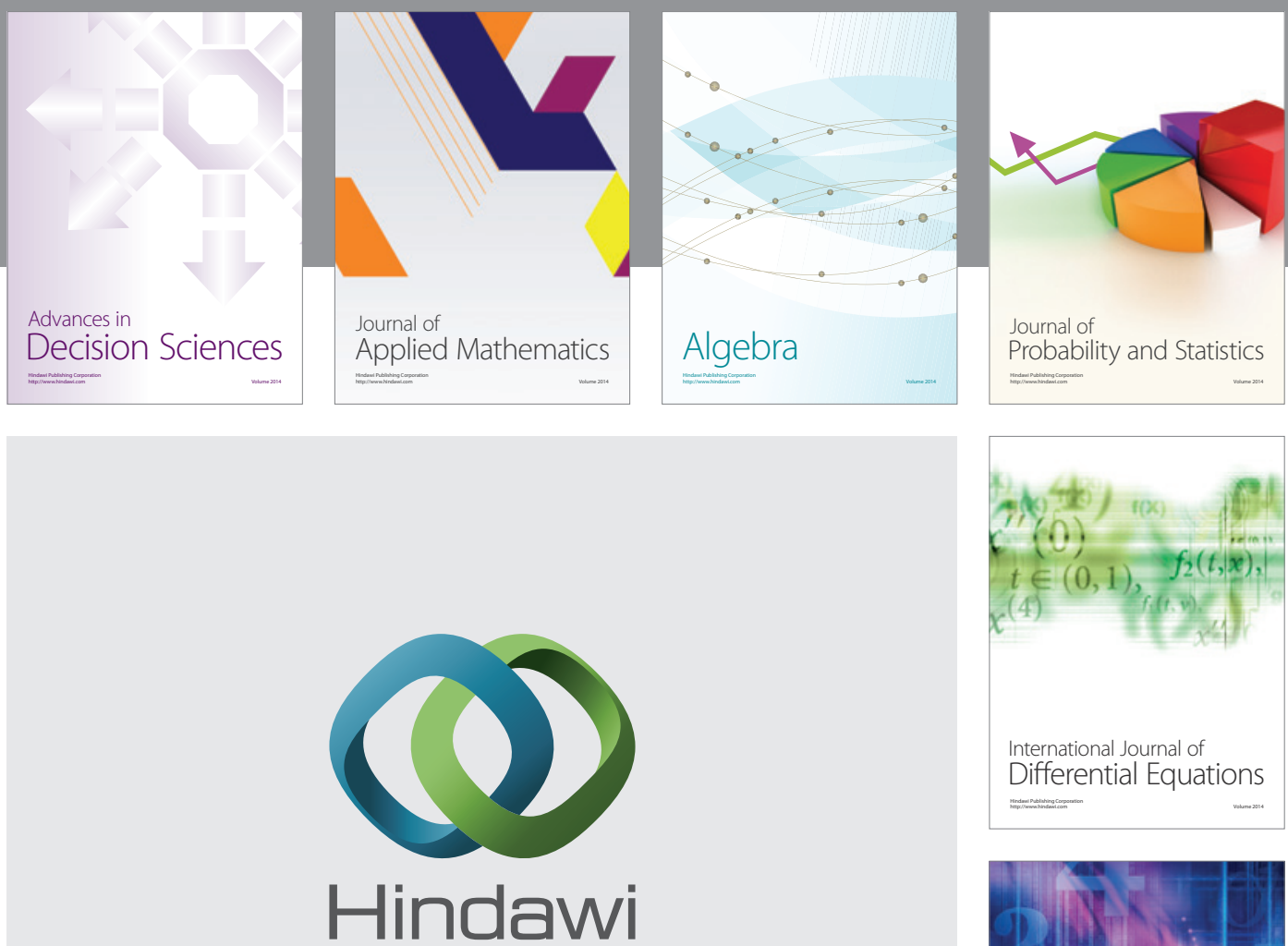

Submit your manuscripts at http://www.hindawi.com
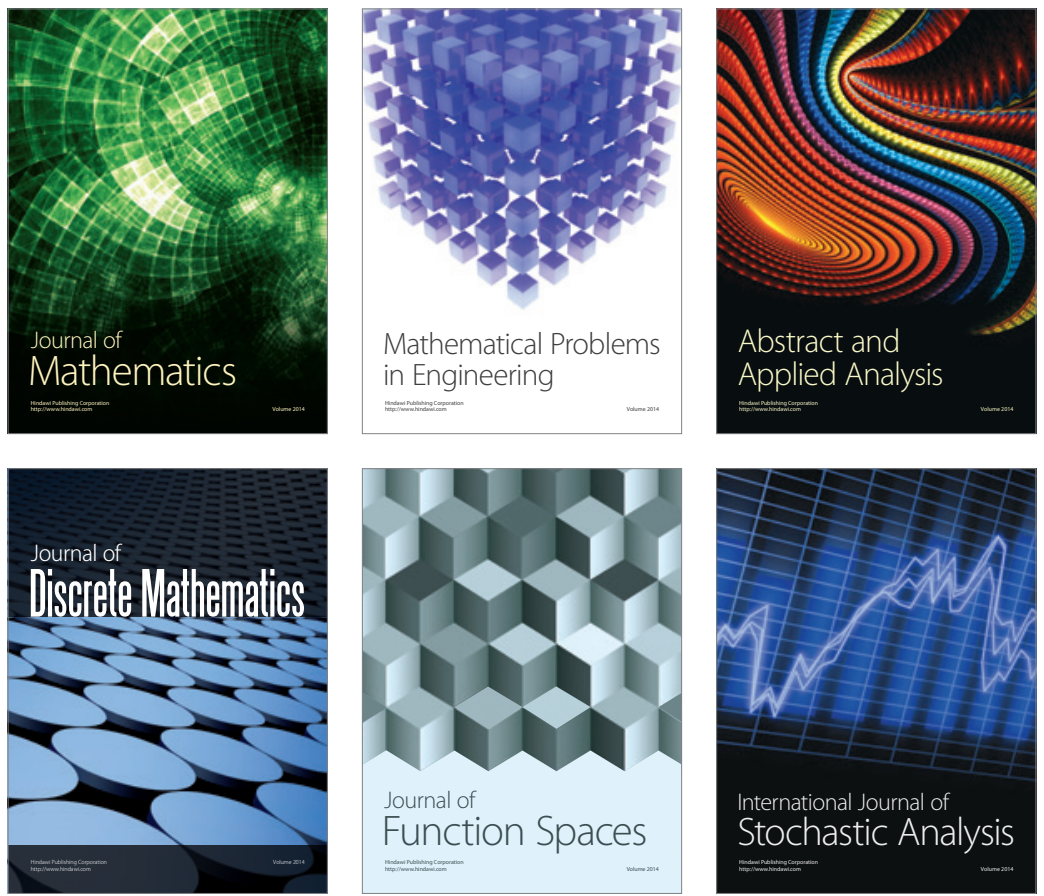

Journal of

Function Spaces

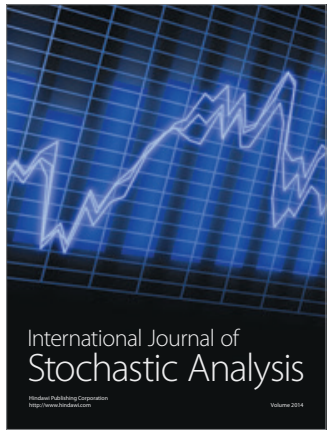

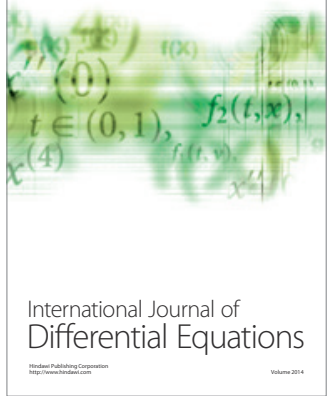
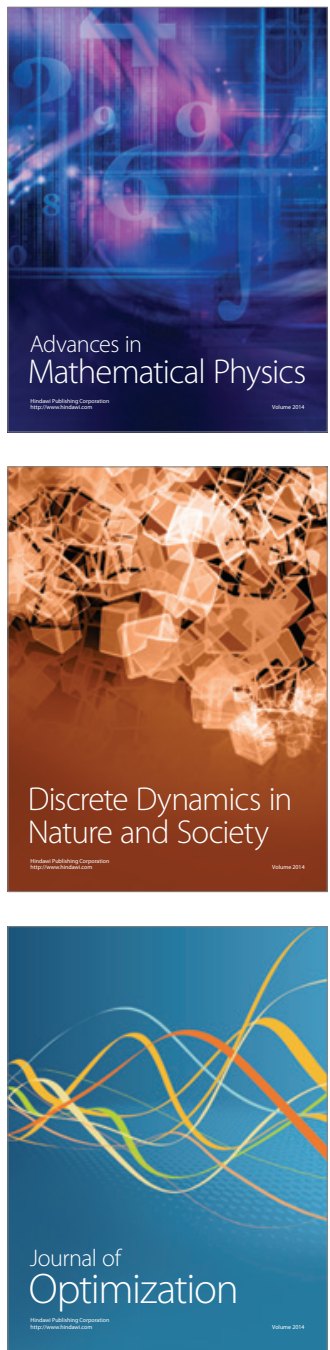